\title{
Estrogen and Mitochondrial Function in Disease
}

\author{
Ved P. Mooga, C. Roger White and \\ Samantha Giordano-Mooga \\ Additional information is available at the end of the chapter
}

http://dx.doi.org/10.5772/intechopen.73015

\begin{abstract}
Anecdotal and scientific evidence suggest that the sex hormone estrogen provides significant health benefits in women. Women have higher estrogen levels than men. Circulating estrogen reaches its highest level during the reproductive period and steadily declines with the onset of menopause. The role of estrogen and estrogen receptors in both cellular physiology and pathophysiology has been controversial. Estrogen has anti-inflammatory and anti-oxidant effects, which preserve cell viability during cardiovascular incidents, but it enhances disease progression in the context of breast cancer. Estrogen mediates these responses via activation of estrogen receptor subtypes located in the cell membrane, nucleus, and mitochondrion. Further, transcription of nuclear and mitochondrial genes by estrogen yields products that play an important role in regulating mitochondrial function. Mitochondria are part of a highly dynamic network and undergo fission and fusion, produce cellular energy, adenosine $5^{\prime}$ triphosphate (ATP), and regulate cell death. Herein, we review the cell and receptor specific effects of estrogen on mitochondrial structure, function, and cell death under normal physiological conditions and in the context of cardiovascular disease, inflammation, neurodegeneration, and cancer. Further research is needed to elucidate the specific role of estrogenic control of mitochondria in health and disease.
\end{abstract}

Keywords: estrogen, mitochondria, aging, menopause, estrogen receptors

\section{Introduction}

The term estrogen refers to a family of chemically similar steroid hormones that include estrone, estradiol, and estriol. Estrogens are synthesized primarily by the ovarian follicles [1]. An important rate limiting step in steroid hormone synthesis is the production of pregnenolone in follicular granulosa cells. Cholesterol is transported from the outer to the inner mitochondrial membrane by the steroidogenic acute regulatory (StAR) protein, followed 
by conversion to pregnenolone by cytochrome P450 side chain cleavage (CYP11A1). Pregnenolone then diffuses to the theca cells where it is converted to androstenedione and then re-routed to the granulosa cells for the aromatase-mediated conversion to estrogen. Androstenedione can also be converted to testosterone. Thus, mitochondria play an important role in estrogen biosynthesis.

Estrogen serves a major role in determining female secondary sex characteristics during development and in regulating the estrous cycle. During puberty and throughout the female reproductive cycle, estrogen levels fluctuate, and as women age, sex steroid production decreases [1]. Estrogen levels oscillate during the estrous cycle. They are lowest during menstruation, steadily rise during the follicular stage and reach a maximal level during ovulation. If a woman becomes pregnant, estrogen levels will remain high, but if fertilization does not occur, hormone levels decline during the luteal phase. Following the luteal phase, menstruation occurs, and the cycle resumes. As estrogen levels fluctuate during the estrous cycle, mitochondria alter the production of pregnenolone accordingly.

Estrogen is a pleiotropic hormone that exerts its effects via both transcriptional and nongenomic mechanisms. In addition to regulating reproductive function, estrogen exerts numerous cytoprotective effects. With respect to atherosclerosis, estrogen regulates levels of circulating lipids by stimulating the formation of high density lipoprotein (HDL) and decreasing expression of low density lipoprotein (LDL) [2]. It exerts antioxidant and anti-inflammatory effects by preventing the oxidation of LDL, inhibiting the expression of endothelial cell adhesion molecules and stimulating nitric oxide formation [3]. As discussed in this chapter, differential responses to estrogen are due to activation of different receptor subtypes. Recent studies also suggest that many of the protective responses to estrogen are related to the ability of the hormone to maintain normal mitochondrial function. Mitochondrial localization of estrogen receptors has been shown to regulate mitochondrial gene expression. In this manner, estrogen plays an important role in the supporting mitochondrial respiration and adenosine $5^{\prime}$ triphosphate (ATP) production, reducing reactive oxygen species (ROS) formation and inhibiting activation of mitochondrial cell death pathways. The goal of this chapter is to discuss mechanisms by which estrogen regulates mitochondrial signaling and function under normal physiological conditions and in the context of disease.

\section{Estrogen receptor types}

Estrogen modulates cellular function via activation of one of four receptor subtypes: estrogen receptor alpha $(E R \alpha)$, estrogen receptor beta $(E R \beta)$, G-protein coupled estrogen receptor (GPER), and ER-X. ER $\alpha$ was first described in the 1950s as a ligand-activated receptor for estrogen, while ER $\beta$ was discovered more recently in 1996 [4]. ERs are located throughout the cell [5]. Two distinct genes encode ligand-activated ER $\alpha$ and ER $\beta$. These genes and their products are subject to epigenetic modifications and alternative RNA splicing [6-11]. Nuclear ER $\alpha$ and ER $\beta$ can modulate gene transcription, while localization of these receptors on the cell membrane results in the rapid activation of signaling cascades via non-genomic mechanisms. 
Studies utilizing ER $\alpha$ and ER $\beta$ knockout $(\mathrm{KO})$ mice have provided insight into the function of each receptor type. Male and female ER $\alpha$ KO mice are infertile, while ER $\beta$ KO mice are fertile but produce small litters [12-14]. These studies highlight the importance of estrogen in the development of reproductive systems of both sexes [14, 15].

$\mathrm{ER} \alpha$ and $\mathrm{ER} \beta$ are also found on the membranes of cellular organelles, including the endoplasmic reticulum and the mitochondrion, where they mediate various cellular functions. Localization to mitochondria was first confirmed using radioligand binding methods in mitochondria isolated from rat uterus and later by immunocytochemistry in rat pancreatic acinar cells [16]. MALDI-TOF mass spectrometry studies have shown that mitochondrial ERs are identical to ERs located in the nucleus [17]. ER $\beta$ is the predominant mitochondrial receptor in most tissues: for example ovary, uterus, spermatocytes, cerebral and hippocampal neurons, cardiomyocytes, and endothelial cells [17, 18]. In contrast, the identification of mitochondrial ER $\alpha$ has been limited to the uterus, ovary, and the MCF-7 breast cancer cell line [18]. The presence of ERs on mitochondria and estrogen responsive elements on the mitochondrial DNA suggests a role for estrogen in regulating the structure and/or function of the organelle [19].

Estrogen also binds to a GPER on the plasma membrane. GPER specifically binds to estradiol and mediates numerous responses including cell proliferation, vasodilation, and regulation of glucose metabolism by non-genomic mechanisms [20]. GPER has also been localized to intracellular sites. In the endoplasmic reticulum, GPER activation induces calcium release and activation of the phosphoinositide 3-kinase (PI3K)-Akt pathway, which induces cell proliferation [21, 22]. While GPER is not associated with the mitochondria, its regulation of cellular calcium handling indirectly impacts mitochondrial function and mitochondrial-induced cell death $[22,23]$. Calcium uptake by mitochondria results in the opening of the mitochondrial permeability transition pore (mPTP) and induction of the intrinsic cell death pathway. GPERspecific agonist G1 binding to GPER has been shown to attenuate these responses in a rodent model of ischemia/reperfusion (I/R) by preventing endoplasmic reticulum calcium release [23]. ER-X is an additional estrogen receptor type that is associated with the cell membrane. This novel receptor shares sequence homology with $\operatorname{ER} \alpha$ and $\operatorname{ER} \beta$, which is expressed primarily in the brain during development and becomes re-expressed in response to ischemic brain injury [24]. While little is known regarding the function of ER-X, some data suggest that it exerts a cytoprotective role in the brain [24].

\section{Mitochondrial function}

The mitochondria are classically described as the powerhouse of the cell by virtue of its ability to generate ATP. Physiological processes underlying mitochondrial bioenergetics and respiration have been previously reviewed [25]. Under aerobic conditions, mitochondria utilize electron transport and a protomotive force to produce $~ 36$ ATP molecules for every glucose molecule. Reducing equivalents produced by the Krebs cycle (NADH and $\mathrm{FADH}_{2}$ ) are accepted by the respiratory chain at Complex I (NADH Dehydrogenase) and Complex 
II (Succinate Dehydrogenase), respectively. Electrons are shuttled through the complexes with oxygen acting as the final electron acceptor at Complex IV. Concurrently, hydrogen ions are pumped from the electronegative mitochondrial matrix into the more positively charged inner membrane space by Complexes I, III (cytochrome C reductase), and IV (cytochrome $\mathrm{C}$ oxidase). The protomotive force thus generated allows hydrogen ions to flow down their concentration gradient at Complex V (ATP Synthase), resulting in the formation of ATP. This chemiosmotic process is tightly regulated and highly efficient. Although ATP is the primary (and often most studied) product of cellular respiration, ROS and thermal energy/heat are also generated by mitochondria. ROS include chemical species produced by the incomplete reduction of $\mathrm{O}_{2}$. These molecules include superoxide anion $\left(\mathrm{O}_{2}{ }^{--}\right)$, hydrogen peroxide $\left(\mathrm{H}_{2} \mathrm{O}_{2}\right)$, and hydroxyl radical $\left({ }^{\circ} \mathrm{OH}\right)$. Mitochondrial ROS are thought to perform a variety of cell signaling functions under normal physiological conditions.

Mitochondria are highly dynamic organelles that are components of a constantly changing, dynamic mitochondrial network. Damaged or old mitochondria can be cleared from the cell by autophagy/mitophagy or bulk clearance and degraded by lysosomal enzymes [26]. Furthermore, mitochondria can also undergo fission and fusion. Fission is the process by which mitochondria bud off from the mitochondrial network. This is regulated by the proteins DRP-1 and FIZZ1. Fusion represents the incorporation of mitochondria in the mitochondrial network and is regulated by mitofusin1 (Mfn1), mitofusin2 (Mfn2), or optic atrophy 1 (OPA1) [27]. These regulatory proteins help to maintain the balance between fission and fusion that is required to preserve cell viability. In different disease states, however, this balance can be disrupted causing mitochondrial dysfunction and cell death.

\section{Mitochondria, cell injury, and apoptosis}

Manganese superoxide dismutase (MnSOD) and glutathione represent endogenous molecules that minimize mitochondrial-derived ROS. Mitochondrial injury occurs, however, when ROS formation exceeds the capacity for their removal by these antioxidant mechanisms. ROS biochemically modify other molecules to produce cytotoxic species that induce cellular injury [28]. For example, ROS induce the formation of 4-hydroxynonenal (HNE), a reactive lipid species that is associated with neuronal damage in brains of Parkinson's disease patients $[29,30]$. ROS production also leads to cell death via the induction of apoptosis. Activation of the intrinsic apoptotic pathway occurs in response to a decrease in mitochondrial membrane potential, opening of the $\mathrm{MPTP}$ and release of cytochrome $\mathrm{C}$ [23]. Cytochrome $C$ initiates the pro-apoptotic cascade by activating the initiator caspase 9 , which in turn cleaves the final effector caspase 3. There are also a number of proteins that regulate apoptosis, including anti-apoptotic Bcl-2, and pro-apoptotic Bax and Bad proteins [31]. When cytosolic Bax binds to the outer mitochondrial membrane, it induces apoptosis by stimulating cytochrome $\mathrm{C}$ release. Further, the binding of Bax to Bcl-2 inhibits the antiapoptotic effects of $\mathrm{Bcl}-2$, resulting in cell death. The dimerization and localization of this group of proteins modulate apoptosis under both basal and pathological conditions and can be modified by the cellular microenvironment. 


\section{Mitochondrial responses to estrogen}

Both nuclear and mitochondrial genes are subject to regulation by estrogen [32]. Nuclear DNA encodes proteins that are incorporated in mitochondria and influence their function. For example, the binding of estrogen to nuclear ER $\alpha$ induces the expression of peroxisome proliferatoractivated receptor gamma coactivator 1-alpha (PGC1 $\alpha)$ [33]. This protein plays an important role in mitochondrial biogenesis, a process by which new mitochondria are formed. The mitochondrion also contains its own maternally inherited DNA, which encodes 37 genes [25]. A recent study in MCF-7 cells showed that estrogen regulates mitochondrial RNA production under serum starvation conditions [34]. Subsequent to estrogen treatment, ER $\alpha$ translocated to the mitochondria and increased expression of mitochondrial tRNAs used to translate mitochondrial proteins. In GH4C1 pituitary cells, treatment with estrogen increases expression of mitochondrial-encoded RNA for subunit II of cytochrome C oxidase [35]. In female rats, levels of mitochondrial-encoded 16S RNA, a housekeeping gene used as a marker for mitochondrial number, are four times higher than males of the same age [36, 37]. Ovariectomy (OVX) in rats is characterized by an increase in liver and brain peroxide production and formation of 8-oxo2'deoxyguanosine, a marker of mitochondrial DNA damage. These changes were associated with a reduction in the antioxidant protein GSH and MnSOD [36]. Estrogen treatment in OVX rats reversed these responses [36]. Collectively, these data suggest that increased estrogen levels regulate mitochondrial and nuclear anti-oxidant protein expression.

Estrogen plays an important role in the regulation of apoptosis by stimulating Bcl-2 protein expression and translocation to the mitochondria. This is achieved via the $\mathrm{Ca}^{2+}$ regulated ERK pathway [38]. The regulation of both Bcl-2 and Bax expression by estrogen has been reported in THP-1 macrophages and human monocyte-derived macrophages. Pre-treatment with estrogen increased the Bcl-2: Bax ratio, thus increasing cell viability in the presence of proapoptotic stimuli. Estrogen treatment of cortical neurons has also been shown to inhibit glutamate toxicity and improve cell viability by upregulating Bcl-2 expression [39]. In SH-SY5Y neuroblastoma cells over-expressing ER $\beta$, the receptor has been shown to interact with the pro-apoptotic protein Bad and prevent its binding to Bax, thereby inhibiting apoptosis [40]. These data suggest that both estrogen and ERs per se are anti-apoptotic and modulate disease pathogenesis. Estrogen also preserves cell viability by altering mitochondrial dynamics. In the myocardium of ischemia reperfusion injury rodents, OVX rodents display an increase in mitochondrial fusion after injury, which is reversed by estrogen treatment [41] Work in isolated cortical astrocytes from male and female postnatal day 1 mice shows that estrogen regulates fission and fusion genes in a gender-specific manner [42]. Further, estrogen stimulated mitochondrial biogenesis in skeletal muscle and adipocytes [43,44]. These data suggest that estrogen can strengthen the mitochondrial network by increasing mitochondrial fusion, thus preserving mitochondrial function and cell viability.

Estrogen effects on mitochondrial function vary in different cell types. For example, estrogen binds specifically to the oligomycin-sensitivity conferring protein of ATP-synthase (Complex V) in brain mitochondria and inhibits ATP production [45]. In contrast, another study showed that the enzymatic activity of F0F1-ATPase, a Complex V subunit, is higher in mitochondria 
isolated from the heart than other tissues. Estrogen induced a further increase in cardiac ATPase activity implying a direct link between estrogen stimulation and ATP production [45, 46]. Estrogen can therefore exert different effects on mitochondria from different cell types. These differential responses may impact disease pathogenesis.

\section{Estrogen, mitochondria, and cardiovascular disease}

Cardiovascular disease (CVD) is the leading cause of death in men and women, and American Heart Association statistics reveal a significant increase in CVD mortality in women compared to men [47]. Mitochondrial dysfunction has been implicated as a causative factor in CVD, with mitochondrial DNA damage being significantly increased in the heart and aorta of patients with CVD compared to healthy controls [25, 48-50]. Women do not generally present with CVD until the seventh decade, while the incidence of death due to CVD is high in men throughout life. This age-dependent increase in CVD in women has been linked to the onset of menopause and a reduction in circulating estrogen levels. The "Free Radical Theory of Aging" proposes that, with increased age, an increase in free radical formation initiates a vicious cycle of ROS formation that causes progressive cell injury [51]. Data suggest that loss of the antioxidant and anti-inflammatory effects of estrogen after menopause contributes to the development of mitochondrial injury $[52,53]$. Thus, maintaining high levels of estrogen may increase lifespan and/or health in postmenopausal women.

Studies using experimental animal models of CVD show that OVX increases vascular inflammation/injury in a manner that is prevented by estrogen treatment [54, 55]. Analysis of mitochondria isolated from hearts of OVX rats reveals increased levels of apoptotic markers compared to mitochondria of intact animals. Administration of estrogen to these animals significantly attenuated apoptosis [56]. Since mitochondrial damage and apoptosis can be mediated by ROS, it has been hypothesized that the estrogen can decrease ROS by activating the antioxidant pathway. Treatment of human aortic endothelial cells (HAECs) with estradiol upregulates the mitochondrial antioxidant MnSOD by an ER $\alpha$-dependent mechanism. The ability of estrogen to increase MnSOD levels is ablated in ER $\alpha \mathrm{KO}$ mice but not in ER $\beta$ KO mice. Interestingly, while ER $\beta$ does not regulate MnSOD expression, it was shown to be essential for preventing atherosclerotic progression in vivo $[57,58]$. These data show that ERs modulate mitochondrial antioxidant production and have distinctive vasoprotective mechanisms.

Gender differences have been identified in mitochondrial genes isolated from rat hearts [59]. Whole genome microarray analysis showed that expression of genes associated with mitochondrial apoptosis pathways is significantly elevated in male mice compared to females. In contrast, genes associated with fatty acid and glucose metabolism were upregulated in females. Female rats also displayed higher transcription levels for mitochondrial Complexes I and IV. These data suggest that genes related to cellular metabolism, including mitochondrial respiration, are upregulated in cardiac mitochondria from female rats while genes associated with mitochondrial apoptosis are increased in males. Whether this difference is directly related to the circulating levels of estrogen in vivo is unclear. 
Mitochondrial structure in the heart is influenced by estrogen. The hearts of OVX rats that underwent I/R injury had lower levels of mitochondrial respiratory function and increased myocardial cell death compared to intact animals. Transmission electron microscopy showed that the mitochondria in cardiomyocytes from OVX rats were more disordered within the cell and structurally damaged compared to mitochondria from intact animals [41]. Interestingly, even male ER $\alpha \mathrm{KO}$ mice that underwent cardiac I/R injury display lower coronary blood flow rates, increased calcium accumulation, and reduced nitrite production compared to non-ischemic hearts. Further, electron microscopic analysis revealed that the mitochondria from ER $\alpha$ KO mice were abnormally shaped [60]. These studies suggest that estrogen signaling plays a role in regulating mitochondrial structure in both females and males. In another model of I/R injury, female wild-type mouse hearts were shown to have better functional recovery and an attenuated inflammatory response compared to female $\mathrm{ER} \alpha \mathrm{KO}$ mice and wild-type male mice [61]. These data further suggest the importance of ER signaling as a cardioprotective mechanism in females.

Effects of estrogen on mitochondrial function have been tested in a genetic model of hypertrophic cardiomyopathy (cTnT-Q92). Estrogen treatment improved ATP production, the mitochondrial respiratory ratio, and diastolic function in OVX cTnT-Q92 mice compared to untreated OVX mice [62]. OVX in CTnT-Q92 mice attenuated the expression of the mitochondrial biogenesis genes PGC1 $\alpha$, peroxisome proliferator-activated receptor alpha (PPAR $\alpha$ ), mitochondrial transcription factor A (tFAM), and the antioxidant protein nuclear respiratory factor 1 (NRF1). Estrogen treatment improved cardiac mitochondrial organization and cristae structure and increased mitochondrial biogenesis. These data directly show that estrogen exerts cytoprotective effects at the level of the mitochondrion that translate into an improvement in cardiac function.

\section{Estrogen, mitochondria, and inflammation}

Macrophages contribute to the chronic inflammation associated with many diseases including CVD and neurodegeneration. Macrophages display plasticity in that they may adopt various phenotypes. The differentiation of these cells is highly dependent on the local microenvironment in which they are situated. M1 macrophages are pro-inflammatory and are induced by cytokines and lipopolysaccharide (LPS). M2 macrophages are anti-inflammatory, play a role in wound healing, and are induced by IL-4 and IL-13 [63, 64]. The metabolic characteristics of M1 and M2 macrophages are different. M1 macrophages rely on glycolysis for ATP formation while M2 macrophages are dependent on mitochondrial oxidative phosphorylation for energy $[63,64]$. Damage to mitochondria induced by inflammatory stimuli can exacerbate cellular injury [65]. It was shown that both $\mathrm{ER} \alpha$ knockout and mitochondrial dysfunction inhibit the IL-4 mediated conversion to macrophages from an M1 to an M2 phenotype [64, 66].

Treatment of macrophages with LPS/interferon- $\gamma(\mathrm{IFN}-\gamma)$ favors an increase in the M1 phenotype. In macrophages isolated from premenopausal women, estrogen treatment was shown to reduce the M1/M2 ratio in cells exposed to LPS/IFN- $\gamma$ to a greater extent than macrophages isolated from postmenopausal women [67]. Recent studies from our group have shown that there is a significant decrease in ER $\alpha$ expression in macrophages from postmenopausal 
women compared to premenopausal women while estrogen therapy was able to preserve ER $\alpha$ expression [68]. These data imply that estrogen and ER levels play a crucial role in macrophage polarization, but the role of estrogen on the mitochondrial function in these groups is still unknown. Determining the role of estrogen on the mitochondria and how it affects macrophage phenotype can help us to better understand the anti-inflammatory roles of estrogen.

\section{Estrogen, mitochondria, and neurodegeneration}

Neurodegenerative disorders such as Alzheimer's disease (AD) and Parkinson's disease (PD) are characterized by the progressive deterioration and death of specific neuron types in the brain [69]. It is well known that mitochondrial dysfunction plays a pathogenic role in neurodegeneration [69]. AD is characterized by atrophy of the cortex and hippocampus. Additionally, increases in glucose metabolism and a reduction in the activities of mitochondrial Complexes I, III, and IV in the brain have been reported $[69,70]$. Plaques containing amyloid beta and tau protein can become incorporated in the remaining brain cells [69]. These inclusions are known to increase oxidative stress and mitochondrial dysfunction.

In PD, the loss of dopaminergic neurons in the substantia nigra contributes to the development of motor disturbances. The protein alpha synuclein becomes entangled with these neurons, resulting in ROS production, mitochondrial dysfunction, and neuronal cell death [71]. A decrease in mitochondrial Complex I activity has been observed in postmortem brains of PD patients that is linked to a reduction in oxidative phosphorylation [72]. In vivo and in vitro models of PD utilize Complex I inhibitors, such as rotenone, to induce PD-like systems to study disease mechanisms [73, 74]. Most PD cases are of unknown etiology; however, data suggest that defects in the expression of the mitochondrial clearance genes parkin, pink1, and DJ-1 may be an underlying mechanism [69].

Gender differences have been identified in the pathology of both AD and PD [75, 76]. These include alterations in brain weight, regional atrophy, distribution of white and gray matter, cerebral blood flow, expression of neurotransmitter transporters and receptors, and age of onset [77]. In the case of PD, there is also an increased incidence of the disease in men compared to women $[78,79]$. The gender difference in neurodegenerative phenotypes has led to the hypothesis that estrogen exerts neuroprotective effects and that these effects are mediated at the level of the mitochondrion [80]. In mouse spinal cord neurons, estrogen treatment increased mRNA levels of nuclear-encoded mitochondrial electron transport chain genes ND1, CytB, Cox2, and ATP6 [80]. ER $\alpha$ has been identified in the mitochondria of endothelial cells in the brain and forebrain. In these cells, estrogen increased expression of cytochrome $\mathrm{C}$ and reduced ROS formation [81]. ER $\beta$ has been localized to hippocampal mitochondria [17, 81, 82]. Hippocampal cells isolated from ER $\beta$ KO mice had lower mitochondrial membrane potential and were more resistant to oxidative stress compared to control mice [83]. Taken together, these data suggest opposing effects of ER $\alpha$ and ER $\beta$ in the brain. More cell-type specific studies are needed to better understand the role of these ERs in the brain and to help clarify these opposing views.

Estrogen also interacts with mitochondrial proteins in a non-genomic manner. Under in vitro conditions, estrogen had no effect on sodium dependent calcium influx from mitochondria isolated from synaptosomes and increased mitochondrial calcium efflux [84]. Thus, estrogen 
prevented mitochondrial calcium overload. Higher levels of cytoplasmic calcium increase mitochondrial ATP production and cause neuron-specific changes in cellular signaling. In aged, post-reproductive rodents, loss of estrogen is associated with a decrease in brain weight and a concomitant increase in the utilization of ketone bodies and fatty acids [85]. This was associated with a decrease in metabolic substrates for mitochondrial ATP production that was further decreased in an AD mouse model [85]. Taken together, we and others propose that reductions in estrogen levels that cause decreased mitochondrial function during the postmenopausal period may explain the increased incidence of $\mathrm{AD}$ in women at this stage.

Estrogen preserves mitochondrial structure/function by upregulating the mitochondrial antioxidant enzyme MnSOD in the brain of female rodents [37, 86, 87]. In SK-N-SH neuroblastoma cells, estrogen inhibits the effects of the mitochondrial Complex II inhibitor, 3-nitroprionic acid (3-NPA), by preserving mitochondrial ATP production and inhibiting the 3-NPA induced hydrogen peroxide and peroxynitrite formation [88]. These data suggest that estrogen also plays an anti-oxidant role in the brain. This has led many to hypothesize that the anti-oxidant effects of estrogen may play a role in slowing disease pathogenesis.

Estrogen also regulates mitochondrial dynamics in astrocytes in a gender-dependent manner. It reduces expression of the fusion protein Mfn1 in astrocytes isolated from male rodents but has no effect on astrocytes obtained from females [80]. Treatment of cortical primary astrocytes with estrogen increases the expression of fission (Dyn 1 and Fis 1) and fusion (Mfn2) proteins to a greater extent in female mice than males. The upregulation of both fission and fusion proteins suggests that mitochondrial network is more dynamic in females than males. Although the exact mechanisms and reasons for the differences in fission and fusion regulation between male and female rodents are unknown, these responses may explain the sexual dimorphism seen in neurodegenerative diseases and other pathologies.

\section{Estrogen, mitochondria, and cancer}

Cancer is the second leading cause of death in the United States, and, among all cancers, breast cancer is the second most commonly diagnosed cancer in women. Breast tumor cells that express estrogen receptors are classified as ER-positive and account for $80 \%$ of all breast cancers [89]. Further, the Women's Health Initiative Study showed that menopausal hormone therapy (MHT) increases the incidence of breast cancer in women compared to controls [90]. Modern day cancer treatment principally focuses on identifying estrogen signatures in breast cancers, and suppression of estrogen receptor function is a routine therapeutic strategy.

Estrogen is known to regulate mitochondrial function in the context of breast cancer by several mechanisms. First, it has been shown to alter mitochondrial morphology. Administration of physiologically relevant doses of estrogen to MCF-7 breast cancer cell lines results in enlargement of mitochondria [91]. Mitochondrial cristae adopt an abnormal structure that is reminiscent of mitochondria that are oxygen-deprived and rely on glycolysis for ATP formation. This change in structure was associated with a 2.5-fold increase in the mitochondrial content of ER $\alpha$ and $\mathrm{ER} \beta$ and an increase in the mitochondrial expression of cytochrome $\mathrm{C}$ oxidase subunits I and II. Alternatively, activation of cell membrane estrogen receptors is reported to induce changes 
in the cytoskeleton that indirectly influences mitochondrial structure [92]. Alteration in mitochondrial structure not only affects the capacity of the energy production but also influences other crucial functions such as calcium signaling, ROS production, or biosynthetic processes.

Second, estrogen induces cellular ROS production in cancer cells by three main pathways: (1) direct inhibition of respiratory chain complexes; (2) accumulation of calcium within mitochondria; and (3) inhibition of the antioxidant response element (ARE) [92]. The increase in ROS generation by the mitochondria and the decrease in antioxidant capacity cause a cellular shift to high ROS production that plays an important role in cancer cell proliferation and cell damage. Estrogen also promotes ROS formation in breast cancer cells by inducing cyclin D1 gene expression [93]. NRF-1 regulates the expression of several nuclear-encoded mitochondrial genes [94] that encode respiratory protein subunits, mtDNA transcription/replication machinery, components of heme biosynthesis, and mitochondrial protein import [95]. Cyclin D1 phosphorylates NRF-1, resulting in repression of its activity. This inactivation of NRF-1 reduces mitochondrial activity and shift glucose metabolism toward glycolysis [96]. The reduction in mitochondrial ATP production, in part, increases mitochondrial ROS production.

Another characteristic of cancer cells is that they highjack apoptotic pathways in order to evade cell death. When normal cells are exposed to UV radiation, the generation of mitochondrial ROS activates c-jun N-terminal kinase (JNK) and protein kinase C (PKC)- $\delta$. These signaling molecules trigger the translocation of Bax to the mitochondria and induce apoptosis. In breast cancer cells exposed to UV radiation, estrogen attenuates cytochrome $C$ release, preserves mitochondrial membrane potential, and inhibits apoptotic cell death [97]. Other data show that addition of estrogen to MCF-7 breast cancer cells induced apoptotic signaling through the extrinsic cell death Fas ligand pathway. This response was accompanied by an increase in the expression of anti-apoptotic Bcl-2 [98].

The role of estrogen receptors in breast cancer development has been known for almost over 30 years. Subsequent studies strongly suggested that ER status is the single most important predictive and prognostic biomarker in breast cancer. Clinicians use several strategies to battle estrogen-sensitive breast cancer, which affect not only estrogen levels but also mitochondrial function. One approach is to block ovarian function. Ovarian ablation can either be performed surgically to remove the ovaries (oophorectomy) or by radiation. An alternative approach is to temporarily suppress the ovarian function pharmacologically using gonadotropin-releasing hormone (GnRH) agonists. GnRH interferes with signals that are produced by the pituitary gland that stimulate the ovaries to produce estrogen. GnRH agonists also act by inducing mitochondrial depolarization, thereby decreasing mitochondrial oxidative capacity. Aromatase inhibitors represent another pharmacological approach to inhibit estrogen synthesis. Addition of aromatase inhibitors to MCF-7 cells was shown to induce caspase 9 expression [99]. Thus, activation of the intrinsic cell death pathway may be an alternative mechanism by which aromatase inhibitors decrease cancer progression. Selective estrogen receptor modulators (SERMs) are another class of drugs that are used for treatment of breast cancers. The SERM tamoxifen blocks the ability of estrogen to stimulate the growth of breast cancer cells. Tamoxifen has also been shown to induce mitochondrial ROS and apoptosis by increasing mitochondrial nitric oxide synthase (mtNOS) [100]. Tamoxifen decreases cellular respiration, increases mitochondrial cytochrome $\mathrm{C}$ release, and increases mitochondrial lipid 
peroxide formation. These data suggest that tamoxifen induces the mitochondrial cell death pathway. While current breast cancer therapeutics inhibit both estrogen signaling and mitochondrial function, the development of next generation drugs that can more efficiently inhibit these processes is required.

\section{Conclusions}

Estrogen is a multi-functional hormone that exerts its effects by both transcriptional and nongenomic mechanisms. Nuclear transcriptional responses are classically induced by binding of estrogen to ER $\alpha$ and ER $\beta$. Estrogen-dependent gene transcription plays an important role in the development of female reproductive structures and regulation of the estrous cycle. The local production of estrogen is now also thought to regulate physiological responses in males. Non-genomic responses to estrogen are more rapid and include induction of signaling pathways that promote cell proliferation. The differential expression of ERs in different cell types and cellular loci dictates their specific function.

Studies in recent years have defined a role for estrogen in the regulation of mitochondrial structure and function. Estrogen increases expression of respiratory complexes, antioxidant molecules, and anti-apoptotic factors that directly impact mitochondrial structure and function. Aging in women is associated with a reduction in estrogen formation and the development of mitochondrial dysfunction. An increase in free radical damage in cells also occurs with aging. Damaged mitochondria are more likely to produce additional ROS, thus initiating a vicious cycle that progressively degrades cellular function. This includes estrogen biosynthesis. Transgenic mice with a mutation in the inner mitochondrial membrane peptidase-2 (IMMP-2) had hyperpolarized mitochondria, which produced increased levels of superoxide and ATP and resulted in impaired ovulation and reduced fertility [101]. Other data show that defective mitochondrial DNA polymerase activity induces mitochondrial dysfunction and infertility in mice [102]. It follows that cytoprotective responses to estrogen at the level of the mitochondrion are ablated. Thus, a reciprocal relationship exists between estrogen and mitochondrial function. Under normal physiological conditions, mitochondria are critical mediators of estrogen biosynthesis and are also targets for estrogen action.

Strong evidence suggests that estrogen plays a major role in promoting the proliferation of both normal and the neoplastic breast cancer cells. However, cancer represents a unique scenario in which estrogen exerts tumorigenic responses in susceptible cells. Prolonged exposure to high levels of estrogen is associated with an increased incidence of breast cancer, which supports models of estrogen-induced carcinogenesis. In breast cancer cells, estrogen is shown to not only stimulate the cell proliferation but also inhibit apoptotic pathways, which can therefore lead to uncontrolled tumor growth. Estrogen increases mitochondrial ROS production that can also promote cancer progression. Further research is needed to expand our understanding of how estrogen induces carcinogenesis.

Estrogen and ER signaling in the mitochondria play an important role in health and disease. We have shown that estrogen effects are cell type and receptor type specific, which explains 
the varied effects of estrogen treatment described in this review. We also understand that despite the protective role of estrogen in inflammation, cardiovascular disease, and neurodegeneration, it promotes breast cancer through both nuclear and mitochondrial regulation. Further research is needed to understand the specific mechanisms of estrogen-induced mitochondrial changes in health and disease.

\section{Acknowledgements}

This work was supported by grants from the National Institutes of Health (GM115367 and DK108836).

\section{Author details}

Ved P. Mooga ${ }^{1}$, C. Roger White ${ }^{2}$ and Samantha Giordano-Mooga ${ }^{3 *}$

*Address all correspondence to: sgiordan@uab.edu

1 GenScript USA Inc., Piscataway, New Jersey, United States

2 Vascular Biology and Hypertension Program, Department of Medicine, University of Alabama at Birmingham, Birmingham, Alabama, United States

3 Clinical and Diagnostic Sciences, School of Health Professions, University of Alabama at Birmingham, Birmingham, Alabama, United States

\section{References}

[1] Velarde MC. Mitochondrial and sex steroid hormone crosstalk during aging. Longevity \& Healthspan. 2014;3(1):2

[2] Fåhraeus L. The effects of estradiol on blood lipids and lipoproteins in postmenopausal women. Obstetrics and Gynecology. 1988;72(5 Suppl):18S-22S

[3] Nathan L, Chaudhuri G. Estrogens and atherosclerosis. Annual Review of Pharmacology and Toxicology. 1997;37:477-515

[4] Dahlman-Wright K, Cavailles V, Fuqua SA, Jordan VC, Katzenellenbogen JA, Korach KS, et al. International Union of Pharmacology. LXIV. Estrogen receptors. Pharmacological Reviews. 2006;58(4):773-781

[5] Levin ER. Integration of the extranuclear and nuclear actions of estrogen. Molecular Endocrinology. 2005;19(8):1951-1959

[6] Zhou J, Cidlowski JA. The human glucocorticoid receptor: One gene, multiple proteins and diverse responses. Steroids. 2005;70(5-7):407-417 
[7] Hirata S, Shoda T, Kato J, Hoshi K. Isoform/variant mRNAs for sex steroid hormone receptors in humans. Trends in Endocrinology and Metabolism. 2003;14(3):124-129

[8] Hirata S, Shoda T, Kato J, Hoshi K. Novel isoforms of the mRNA for human female sex steroid hormone receptors. The Journal of Steroid Biochemistry and Molecular Biology. 2002;83(1-5):25-30

[9] Lewandowski S, Kalita K, Kaczmarek L. Estrogen receptor beta. Potential functional significance of a variety of mRNA isoforms. FEBS Letters. 2002;524(1-3):1-5

[10] Post WS, Goldschmidt-Clermont PJ, Wilhide CC, Heldman AW, Sussman MS, Ouyang P, et al. Methylation of the estrogen receptor gene is associated with aging and atherosclerosis in the cardiovascular system. Cardiovascular Research. 1999;43(4):985-991

[11] Ying AK, Hassanain HH, Roos CM, Smiraglia DJ, Issa JJ, Michler RE, et al. Methylation of the estrogen receptor-alpha gene promoter is selectively increased in proliferating human aortic smooth muscle cells. Cardiovascular Research. 2000;46(1):172-179

[12] Couse JF, Korach KS. Estrogen receptor null mice: What have we learned and where will they lead us? Endocrine Reviews. 1999;20(3):358-417

[13] Dupont S, Krust A, Gansmuller A, Dierich A, Chambon P, Mark M. Effect of single and compound knockouts of estrogen receptors alpha (ERalpha) and beta (ERbeta) on mouse reproductive phenotypes. Development. 2000;127(19):4277-4291

[14] Krege JH, Hodgin JB, Couse JF, Enmark E, Warner M, Mahler JF, et al. Generation and reproductive phenotypes of mice lacking estrogen receptor beta. Proceedings of the National Academy of Sciences of the United States of America. 1998;95(26):15677-15682

[15] Luconi M, Forti G, Baldi E. Genomic and nongenomic effects of estrogens: Molecular mechanisms of action and clinical implications for male reproduction. The Journal of Steroid Biochemistry and Molecular Biology. 2002;80(4-5):369-381

[16] Noteboom WD, Gorski J. Stereospecific binding of estrogens in the rat uterus. Archives of Biochemistry and Biophysics. 1965;111(3):559-568

[17] Yang SH, Liu R, Perez EJ, Wen Y, Stevens SM, Valencia T, et al. Mitochondrial localization of estrogen receptor beta. Proceedings of the National Academy of Sciences of the United States of America. 2004;101(12):4130-4135

[18] Psarra AM, Sekeris CE. Steroid and thyroid hormone receptors in mitochondria. IUBMB Life. 2008;60(4):210-223

[19] Chen JQ, Eshete M, Alworth WL, Yager JD. Binding of MCF-7 cell mitochondrial proteins and recombinant human estrogen receptors alpha and beta to human mitochondrial DNA estrogen response elements. Journal of Cellular Biochemistry. 2004;93(2):358-373

[20] Sharma G, Mauvais-Jarvis F, Prossnitz ER. Roles of G protein-coupled estrogen receptor GPER in metabolic regulation. The Journal of Steroid Biochemistry and Molecular Biology. 2018;176(1879-1220):31-37

[21] Revankar CM, Cimino DF, Sklar LA, Arterburn JB, Prossnitz ER. A transmembrane intracellular estrogen receptor mediates rapid cell signaling. Science. 2005;307(5715):1625-1630 
[22] Prossnitz ER, Barton M. The G-protein-coupled estrogen receptor GPER in health and disease. Nature Reviews. Endocrinology. 2011;7(12):715-726

[23] Bopassa JC, Eghbali M, Toro L, Stefani E. A novel estrogen receptor GPER inhibits mitochondria permeability transition pore opening and protects the heart against ischemiareperfusion injury. American Journal of Physiology. Heart and Circulatory Physiology. 2010;298(1):H16-H23

[24] Toran-Allerand CD, Guan X, MacLusky NJ, Horvath TL, Diano S, Singh M, et al. ER-X: A novel, plasma membrane-associated, putative estrogen receptor that is regulated during development and after ischemic brain injury. The Journal of Neuroscience. 2002;22(19):8391-8401

[25] Ballinger SW. Mitochondrial dysfunction in cardiovascular disease. Free Radical Biology \& Medicine. 2005;38(10):1278-1295

[26] Zhang J. Autophagy and mitophagy in cellular damage control. Redox Biology. 2013; 1(1):19-23

[27] Archer SL. Mitochondrial dynamics - mitochondrial fission and fusion in human diseases. The New England Journal of Medicine. 2013;369(23):2236-2251

[28] D'Autréaux B, Toledano MB. ROS as signalling molecules: Mechanisms that generate specificity in ROS homeostasis. Nature Reviews. Molecular Cell Biology. 2007; 8(10):813-824

[29] YoritakaA,HattoriN, Uchida K, Tanaka M,Stadtman ER, Mizuno Y.Immunohistochemical detection of 4-hydroxynonenal protein adducts in Parkinson disease. Proceedings of the National Academy of Sciences of the United States of America. 1996;93(7):2696-2701

[30] Perluigi M, Coccia R, Butterfield A. HNE a reactive product of lipid peroxidation and neurodegenerative diseases: A toxic combination illuminated by Redox proteomics studies. Antioxidants \& Redox Signaling 2012;11(1557-7716):1590-609

[31] Czabotar PE, Lessene G, Strasser A, Adams JM. Control of apoptosis by the BCL-2 protein family: Implications for physiology and therapy. Nature Reviews. Molecular Cell Biology. 2014;15(1):49-63

[32] Demonacos C, Djordjevic-Markovic R, Tsawdaroglou N, Sekeris CE. The mitochondrion as a primary site of action of glucocorticoids: The interaction of the glucocorticoid receptor with mitochondrial DNA sequences showing partial similarity to the nuclear glucocorticoid responsive elements. The Journal of Steroid Biochemistry and Molecular Biology. 1995;55(1):43-55

[33] Schreiber SN, Emter R, Hock MB, Knutti D, Cardenas J, Podvinec M, et al. The estrogenrelated receptor alpha (ERRalpha) functions in PPARgamma coactivator 1alpha (PGC1alpha)-induced mitochondrial biogenesis. Proceedings of the National Academy of Sciences of the United States of America. 2004;101(17):6472-6477

[34] Sanchez MI, Shearwood AM, Chia T, Davies SM, Rackham O, Filipovska A. Estrogenmediated regulation of mitochondrial gene expression. Molecular Endocrinology. 2015; 29(1):14-27 
[35] Van Itallie CM, Dannies PS. Estrogen induces accumulation of the mitochondrial ribonucleic acid for subunit II of cytochrome oxidase in pituitary tumor cells. Molecular Endocrinology. 1988;2(4):332-337

[36] Borrás C, Sastre J, García-Sala D, Lloret A, Pallardó FV, Viña J. Mitochondria from females exhibit higher antioxidant gene expression and lower oxidative damage than males. Free Radical Biology \& Medicine. 2003;34(5):546-552

[37] Borrás C, Gambini J, Gómez-Cabrera MC, Sastre J, Pallardó FV, Mann GE, et al. 17betaoestradiol up-regulates longevity-related, antioxidant enzyme expression via the ERK1 and ERK2[MAPK]/NFkappaB cascade. Aging Cell. 2005;4(3):113-118

[38] Subramanian M, Shaha C. Up-regulation of Bcl-2 through ERK phosphorylation is associated with human macrophage survival in an estrogen microenvironment. Journal of Immunology. 2007;179(4):2330-2338

[39] Nilsen J, Diaz Brinton R. Mechanism of estrogen-mediated neuroprotection: Regulation of mitochondrial calcium and Bcl-2 expression. Proceedings of the National Academy of Sciences of the United States of America. 2003;100(5):2842-2847

[40] Liang J, Xie Q, Li P, Zhong X, Chen Y. Mitochondrial estrogen receptor $\beta$ inhibits cell apoptosis via interaction with bad in a ligand-independent manner. Molecular and Cellular Biochemistry. 2015;401(1-2):71-86

[41] Zhai P, Eurell TE, Cotthaus R, Jeffery EH, Bahr JM, Gross DR. Effect of estrogen on global myocardial ischemia-reperfusion injury in female rats. American Journal of Physiology. Heart and Circulatory Physiology. 2000;279(6):H2766-H2775

[42] Arnold S, de Araújo GW, Beyer C. Gender-specific regulation of mitochondrial fusion and fission gene transcription and viability of cortical astrocytes by steroid hormones. Journal of Molecular Endocrinology. 2008;41(5):289-300

[43] Capllonch-Amer G, Sbert-Roig M, Galmés-Pascual BM, Proenza AM, Lladó I, Gianotti M, et al. Estradiol stimulates mitochondrial biogenesis and adiponectin expression in skeletal muscle. The Journal of Endocrinology. 2014;221(3):391-403

[44] Capllonch-Amer G, Lladó I, Proenza AM, García-Palmer FJ, Gianotti M. Opposite effects of 17- $\beta$ estradiol and testosterone on mitochondrial biogenesis and adiponectin synthesis in white adipocytes. Journal of Molecular Endocrinology. 2014;52(2):203-214

[45] Zheng J, Ramirez VD. Purification and identification of an estrogen binding protein from rat brain: Oligomycin sensitivity-conferring protein (OSCP), a subunit of mitochondrial F0F1-ATP synthase/ATPase. The Journal of Steroid Biochemistry and Molecular Biology. 1999;68(1-2):65-75

[46] Kipp JL, Ramirez VD. Effect of estradiol, diethylstilbestrol, and resveratrol on F0F1ATPase activity from mitochondrial preparations of rat heart, liver, and brain. Endocrine. 2001;15(2):165-175

[47] Benjamin EJ, Blaha MJ, Chiuve SE, Cushman M, Das SR, Deo R, et al. Heart disease and stroke Statistics-2017 update: A report from the American Heart Association. Circulation. 2017;135(10):e146-e603 
[48] Corral-Debrinski M, Shoffner JM, Lott MT, Wallace DC. Association of mitochondrial DNA damage with aging and coronary atherosclerotic heart disease. Mutation Research. 1992;275(3-6):169-180

[49] Corral-Debrinski M, Stepien G, Shoffner JM, Lott MT, Kanter K, Wallace DC. Hypoxemia is associated with mitochondrial DNA damage and gene induction. Implications for cardiac disease. Journal of the American Medical Association. 1991;266(13):1812-1816

[50] Ballinger SW, Patterson C, Knight-Lozano CA, Burow DL, Conklin CA, Hu Z, et al. Mitochondrial integrity and function in atherogenesis. Circulation. 2002;106(5):544-549

[51] Harman D. Aging: A theory based on free radical and radiation chemistry. Journal of Gerontology. 1956;11(3):298-300

[52] Klinge CM. Estrogenic control of mitochondrial function and biogenesis. Journal of Cellular Biochemistry. 2008;105(6):1342-1351

[53] Klinge CM. Estrogens regulate life and death in mitochondria. Journal of Bioenergetics and Biomembranes. 2017;49(4):307-324

[54] Bowling MR, Xing D, Kapadia A, Chen YF, Szalai AJ, Oparil S, et al. Estrogen effects on vascular inflammation are age dependent: Role of estrogen receptors. Arteriosclerosis, Thrombosis, and Vascular Biology. 2014;34(7):1477-1485

[55] Xing D, Nozell S, Chen YF, Hage F, Oparil S. Estrogen and mechanisms of vascular protection. Arteriosclerosis, Thrombosis, and Vascular Biology. 2009;29(3):289-295

[56] Liou CM, Yang AL, Kuo CH, Tin H, Huang CY, Lee SD. Effects of 17beta-estradiol on cardiac apoptosis in ovariectomized rats. Cell Biochemistry and Function. 2010;28(6):521-528

[57] Liu Z, Gou Y, Zhang H, Zuo H, Yao D. Estradiol improves cardiovascular function through up-regulation of SOD2 on vascular wall. Redox Biology. 2014;3:88-99

[58] Zhao C, Dahlman-Wright K, Gustafsson J. Estrogen signaling via estrogen receptor \{beta\}. The Journal of Biological Chemistry. 2010;285(51):39575-39579

[59] Vijay V, Han T, Moland CL, Kwekel JC, Fuscoe JC, Desai VG. Sexual dimorphism in the expression of mitochondria-related genes in rat heart at different ages. PLoS One. 2015;10(1):e0117047

[60] Zhai P, Eurell TE, Cooke PS, Lubahn DB, Gross DR. Myocardial ischemia-reperfusion injury in estrogen receptor-alpha knockout and wild-type mice. American Journal of Physiology. Heart and Circulatory Physiology. 2000;278(5):H1640-H1647

[61] Wang M, Crisostomo P, Wairiuko GM, Meldrum DR. Estrogen receptor-alpha mediates acute myocardial protection in females. American Journal of Physiology. Heart and Circulatory Physiology. 2006;290(6):H2204-H2209

[62] Chen Y, Zhang Z, Hu F, Yang W, Yuan J, Cui J, et al. 17ß-estradiol prevents cardiac diastolic dysfunction by stimulating mitochondrial function: A preclinical study in a mouse model of a human hypertrophic cardiomyopathy mutation. The Journal of Steroid Biochemistry and Molecular Biology. 2015;147:92-102 
[63] Odegaard JI, Chawla A. Alternative macrophage activation and metabolism. Annual Review of Pathology. 2011;6:275-297

[64] Bolego C, Cignarella A, Staels B, Chinetti-Gbaguidi G. Macrophage function and polarization in cardiovascular disease: A role of estrogen signaling? Arteriosclerosis, Thrombosis, and Vascular Biology. 2013;33(6):1127-1134

[65] López-Armada MJ, Riveiro-Naveira RR, Vaamonde-García C, Valcárcel-Ares MN. Mitochondrial dysfunction and the inflammatory response. Mitochondrion. 2013; 13(2):106-118

[66] Van den Bossche J, Neele AE, Hoeksema MA, de Winther MP. Macrophage polarization: The epigenetic point of view. Current Opinion in Lipidology. 2014;25(5):367-373

[67] Toniolo A, Fadini GP, Tedesco S, Cappellari R, Vegeto E, Maggi A, et al. Alternative activation of human macrophages is rescued by estrogen treatment in vitro and impaired by menopausal status. The Journal of Clinical Endocrinology and Metabolism. 2015;100(1):E50-E58

[68] Hage FG, Xing D, Guo Y, Colon C, Szalai AJ, Chen YF, Oparil S. Abstract 14875: Estrogeninduced vasoprotection is preserved after prolonged estrogen deprivation. Circulation. 2014;130:A14875

[69] Johri A, Beal MF. Mitochondrial dysfunction in neurodegenerative diseases. The Journal of Pharmacology and Experimental Therapeutics. 2012;342(3):619-630

[70] Trimmer PA, Swerdlow RH, Parks JK, Keeney P, Bennett JP, Miller SW, et al. Abnormal mitochondrial morphology in sporadic Parkinson's and Alzheimer's disease cybrid cell lines. Experimental Neurology. 2000;162(1):37-50

[71] Schapira AH, Gegg M. Mitochondrial contribution to Parkinson's disease pathogenesis. Parkinson's Disease. 2011;2011:159160

[72] Parker WD, Parks JK, Swerdlow RH. Complex I deficiency in Parkinson's disease frontal cortex. Brain Research. 2008;1189:215-218

[73] Giordano S, Lee J, Darley-Usmar VM, Zhang J. Distinct effects of rotenone, 1-methyl4-phenylpyridinium and 6-hydroxydopamine on cellular bioenergetics and cell death. PLoS One. 2012;7(9):e44610

[74] Greenamyre JT, Cannon JR, Drolet R, Mastroberardino PG. Lessons from the rotenone model of Parkinson's disease. Trends in Pharmacological Sciences. 2010;31(4):141-142 author reply 2-3

[75] Mazure CM, Swendsen J. Sex differences in Alzheimer's disease and other dementias. Lancet Neurology. 2016;15(5):451-452

[76] Gillies GE, Pienaar IS, Vohra S, Qamhawi Z. Sex differences in Parkinson's disease. Frontiers in Neuroendocrinology. 2014;35(3):370-384

[77] Cosgrove KP, Mazure CM, Staley JK. Evolving knowledge of sex differences in brain structure, function, and chemistry. Biological Psychiatry. 2007;62(8):847-855 
[78] Swerdlow RH, Parker WD, Currie LJ, Bennett JP, Harrison MB, Trugman JM, et al. Gender ratio differences between Parkinson's disease patients and their affected relatives. Parkinsonism \& Related Disorders. 2001;7(2):129-133

[79] Taylor KS, Cook JA, Counsell CE. Heterogeneity in male to female risk for Parkinson's disease. Journal of Neurology, Neurosurgery, and Psychiatry. 2007;78(8):905-906

[80] Arnold S, Victor MB, Beyer C. Estrogen and the regulation of mitochondrial structure and function in the brain. The Journal of Steroid Biochemistry and Molecular Biology. 2012;131(1-2):2-9

[81] Razmara A, Sunday L, Stirone C, Wang XB, Krause DN, Duckles SP, et al. Mitochondrial effects of estrogen are mediated by estrogen receptor alpha in brain endothelial cells. The Journal of Pharmacology and Experimental Therapeutics. 2008;325(3):782-790

[82] Alvarez-Delgado C, Mendoza-Rodríguez CA, Picazo O, Cerbón M. Different expression of alpha and beta mitochondrial estrogen receptors in the aging rat brain: Interaction with respiratory complex V. Experimental Gerontology. 2010;45(7-8):580-585

[83] Yang SH, Sarkar SN, Liu R, Perez EJ, Wang X, Wen Y, et al. Estrogen receptor beta as a mitochondrial vulnerability factor. The Journal of Biological Chemistry. 2009; 284(14):9540-9548

[84] Horvat A, Petrović S, Nedeljković N, Martinović JV, Nikezić G. Estradiol affect $\mathrm{Na}$-dependent $\mathrm{Ca}^{2+}$ efflux from synaptosomal mitochondria. General Physiology and Biophysics. 2000;19(1):59-71

[85] Yao J, Hamilton RT, Cadenas E, Brinton RD. Decline in mitochondrial bioenergetics and shift to ketogenic profile in brain during reproductive senescence. Biochimica et Biophysica Acta. 2010;1800(10):1121-1126

[86] Simpkins JW, Yi KD, Yang SH, Dykens JA. Mitochondrial mechanisms of estrogen neuroprotection. Biochimica et Biophysica Acta. 2010;1800(10):1113-1120

[87] Razmara A, Duckles SP, Krause DN, Procaccio V. Estrogen suppresses brain mitochondrial oxidative stress in female and male rats. Brain Research. 2007;1176:71-81

[88] Wang J, Green PS, Simpkins JW. Estradiol protects against ATP depletion, mitochondrial membrane potential decline and the generation of reactive oxygen species induced by 3-nitroproprionic acid in SK-N-SH human neuroblastoma cells. Journal of Neurochemistry. 2001;77(3):804-811

[89] Kohler BA, Sherman RL, Howlader N, Jemal A, Ryerson AB, Henry KA, et al. Annual report to the nation on the status of cancer, 1975-2011, featuring incidence of breast cancer subtypes by race/ethnicity, poverty, and state. Journal of the National Cancer Institute. 2015;107(6):djv048

[90] Anderson GL, Limacher M, Assaf AR, Bassford T, Beresford SA, Black H, et al. Effects of conjugated equine estrogen in postmenopausal women with hysterectomy: The Women's Health Initiative randomized controlled trial. Journal of the American Medical Association. 2004;291(14):1701-1712 
[91] Vic P, Vignon F, Derocq D, Rochefort H. Effect of estradiol on the ultrastructure of the MCF7 human breast cancer cells in culture. Cancer Research. 1982;42(2):667-673

[92] Felty Q, Roy D. Estrogen, mitochondria, and growth of cancer and non-cancer cells. Journal of Carcinogenesis. 2005;4(1):1

[93] Castro-Rivera E, Samudio I, Safe S. Estrogen regulation of cyclin D1 gene expression in ZR-75 breast cancer cells involves multiple enhancer elements. The Journal of Biological Chemistry. 2001;276(33):30853-30861

[94] Scarpulla RC. Nuclear control of respiratory gene expression in mammalian cells. Journal of Cellular Biochemistry. 2006;97(4):673-683

[95] Cam H, Balciunaite E, Blais A, Spektor A, Scarpulla RC, Young R, et al. A common set of gene regulatory networks links metabolism and growth inhibition. Molecular Cell. 2004;16(3):399-411

[96] Cuezva JM, Krajewska M, de Heredia ML, Krajewski S, Santamaría G, Kim H, et al. The bioenergetic signature of cancer: A marker of tumor progression. Cancer Research. 2002;62(22):6674-6681

[97] Pedram A, Razandi M, Wallace DC, Levin ER. Functional estrogen receptors in the mitochondria of breast cancer cells. Molecular Biology of the Cell. 2006;17(5):2125-2137

[98] Song RX, Zhang Z, Mor G, Santen RJ. Down-regulation of Bcl-2 enhances estrogen apoptotic action in long-term estradiol-depleted ER(+) breast cancer cells. Apoptosis. 2005; 10(3):667-678

[99] Amaral C, Borges M, Melo S, da Silva ET, Correia-da-Silva G, Teixeira N. Apoptosis and autophagy in breast cancer cells following exemestane treatment. PLoS One. 2012; 7(8):e42398

[100] Nazarewicz RR, Zenebe WJ, Parihar A, Larson SK, Alidema E, Choi J, et al. Tamoxifen induces oxidative stress and mitochondrial apoptosis via stimulating mitochondrial nitric oxide synthase. Cancer Research. 2007;67(3):1282-1290

[101] Lu B, Poirier C, Gaspar T, Gratzke C, Harrison W, Busija D, et al. A mutation in the inner mitochondrial membrane peptidase 2-like gene (Immp2l) affects mitochondrial function and impairs fertility in mice. Biology of Reproduction. 2008;78(4):601-610

[102] Trifunovic A, Wredenberg A, Falkenberg M, Spelbrink JN, Rovio AT, Bruder CE, et al. Premature ageing in mice expressing defective mitochondrial DNA polymerase. Nature. 2004;429(6990):417-423 
